\title{
Extended Shadowing Compensation Model in Robust SEM Photometric Stereo*
}

\author{
Deshan $\mathrm{CHEN}^{* *}$ Atsushi MIYAMOTO*** Shun'ichi KANEKO ${ }^{\dagger}$
}

\begin{abstract}
This paper presents an extended shadowing compensation model for eliminating shadowing errors in the scanning electron microscope photometric stereo method. This extended model removes the earlier restrictive assumption that the shadowing effects exist only on horizontal surfaces. This model is developed by modeling image intensities in both the absence and presence of shadowing. A numerical solution is proposed to resolve the implicit expressions for double integrals in the model. Compared to the previous shadowing compensation model, the extended model can be more accurate when applied to shadowing regions on surfaces having large inclinations.
\end{abstract}

Key words: scanning electron microscope (SEM), surface reconstruction, shadowing compensation, backscattering electron (BSE), SEM photometric stereo

\section{Introduction}

The scanning electron microscope (SEM) is a very important tool for observing micro-structure which is in part benefit from its high resolution, and has been widely used in the areas of medical observation, semiconductors, material analysis, etc. The need for three dimensional (3D) surface measurements is significantly increasing. For instance, 3D surface information can provide valuable clues for inspecting and analyzing defects in products of semiconductor manufacturing processes, such as recognition of device patterns and defect regions, classification of defects in terms of the processes responsible for their generation (surface/embedded defects ${ }^{1)}$ ) and the impact on electric properties (volume or shorting/disconnection defect), and specification and control of the issue process, etc. However, SEM can only produce 2D-images directly, and 3D shape measurement in SEM remains a very challenging task.

Attempts have been made to transform SEM into a 3D measuring tool for almost 30 years, and scientific research on 3D surface reconstruction in SEM continues to be intensively studied. Different approaches, which can be broadly classified into two groups, have been utilized. One popular group is generally referred to as the "stereometric" method ${ }^{2-4)}$, in which a pair of stereo images of a specimen at different inclination angles are taken, and the height or depth is determined by measuring the deviations of corresponding points on the two images. However, this method can only be applied to the measurements of surface points where recognizable fine structures exist. In general, it cannot be used on smooth surfaces or to reconstruct a continuous surface profile at high magnification.

The second group of approaches is the so-called "photometric stereo" method ${ }^{5-9)}$, in which multiple secondary or backscattered electron detectors (generally two or four) are symmetrically

$*$ 原稿受付 平成 25 年 5 月 1 日

** 北海道大学大学院情報科学研究科

*** 正会員 (株) 日立製作所横浜研究所（神奈川県横浜市

戸塚区吉田町 292)

†正会員 北海道大学大学院情報科学研究科（札幌市北区

北 14 条西 $9 丁$ 目) positioned about the beam axis, so that the gradient information on the surface being observed can be estimated from multiple images that are measured simultaneously by various detectors. The 3D surface is consequently obtained from the gradient information by an integration scheme. However, these studies have focused almost exclusively on deriving accurate gradient measurement formulas, i.e., expressions of the relationships between the gradient component(s) of target objects and the measured SEM signals from multiple detectors at the points of observation, in which shape information from the surrounding area is excluded. Signal values are generally influenced by shadowing effects associated with the surrounding topography, because such effects absorb a portion of the electrons and are the main source of errors in 3D measurements.

Paluszyński and Slówko ${ }^{10)}$ introduced several techniques for reconstructing the shape in the shadowed region that utilize two or three unshadowed detectors under a setting of four secondary electron (SE) detectors. However, this method requires exact geometric parameters for the detector system, which are generally difficult to obtain. In addition, shadowed regions must be manually identified from the SE images each time, which is restrictive for some actual applications.

The present authors have introduced a shadowing compensation model with which both an accurate 3D surface and a compensated shadowless images can be obtained after convergence of an iterative shadowing compensation method ${ }^{11}$. The shadowing compensation model was derived by modeling the mechanism that generates shadowing effects; i.e., we modeled signal outputs in both the absence and presence of shadowing. In the theoretical derivation of the model, we assumed that shadowing effects exist only in the horizontal region. This assumption simplifies the model and introduces certain benefits, such as compact explicit solutions, convenient calculations. However, when the model is applied to shadowing regions on surfaces with sufficiently large inclinations, the resulting errors are large.

To increase accuracy and expand the scope of the shadowing compensation model, we extend the model from horizontal surfaces to general inclined surfaces. Analogous to the previous sce- 


\section{BSE \\ Detector 1}

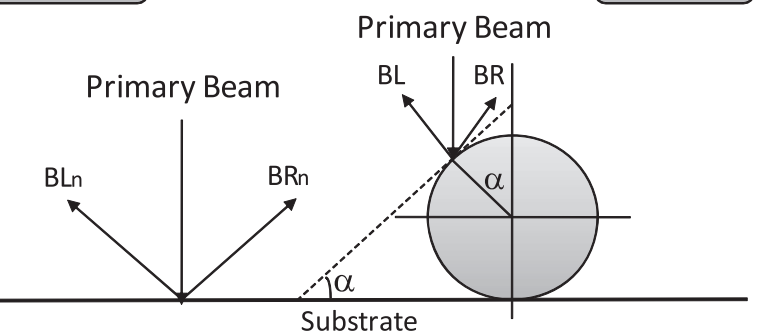

Substrate

Fig. 1: Schematic diagram of measuring system with two BSE detectors

nario in the horizontal case, the extended shadowing compensation model is derived by modeling the shadowing generation process, i.e., the image intensity in both the absence and presence of shadowing, while abandoning the restrictive assumption that shadowing effects exist only on horizontal surfaces. Unlike the previous model, the extended shadowing compensation model does not have a closed-form solution. Hence, we explore a numerical approach that is also computationally efficient. The two main contributions of this study are as follows: (1) We extend the shadowing compensation model from horizontal surfaces to general inclined surfaces. (2) We present numerical solutions for the extended shadowing compensation model.

\section{Idea of the Shadowing Compensation Method}

For a successful implementation of SEM photometric stereo method, two backscattering electron (BSE) detectors are equipped in our measuring system (as shown in Fig. 1). The two detectors are oppositely positioned along the $x$-axis, which can simultaneously provide two BSE images from the two detectors, denoted as $B L$ and $B R$, respectively. In general, we use $B L$ and $B R$ to refer to the left and right image and detector 1 and 2 to designate the left and right detector, respectively. Suganuma ${ }^{7)}$ proposed an experimentally derived equation that could measure the gradient component in the direction of the $x$-axis:

$$
\frac{\partial z}{\partial x}=k \frac{B L^{2}-B R^{2}}{\left(B L_{n}+B R_{n}\right)^{2}},
$$

where $k$ is a constant and $B L_{n}$ and $B R_{n}$ are signal outputs from two detectors on the horizontal specimen surface. Finally, the surface profile can be reconstructed by some depth from gradient method, such as numerical integration method, optimization method, etc. (the optimization method can generally be more robust against noise influence ${ }^{11-13)}$ ).

However, gradient measurement formula (1) does not take the shape information from the surrounding area of the points of the observation. In fact, shadowing phenomena can frequently occur in imaging processes owing to the exceptional features of sample topography. As shown in Fig. 2, a portion of the electrons is screened out because of occlusion, resulting in fewer electrons to be collected by the BSE detector, which consequently gives rise to smaller observed image intensity than the underlying shadowless one. Therefore, the gradient measurements generally contain large deviations when applying model (1) in a region with shadowing effects. It is thus obvious that shadowing effects can contribute significantly to the final error of a reconstructed 3D surface if only model (1) is employed.

To address the shadowing issue, we have introduced a shadowing compensation model ${ }^{11)}$ that provides an important cue for estimating the underlying shadowless images from observed images with shadowing effects. With such a model, we can estimate the underlying shadowless image intensity from the observed one, provided the corresponding shadowing angles are known. The dilemma is that the true shadowing angles are determined by the true 3D surface, which is initially unknown. We propose to solve this problem in an iterative scheme that performs commutatively between compensation of image intensities and modification of the corresponding 3D surface. Both the reconstructed 3D surface and compensated shadowless images can be obtained after convergence of the method.

The shadowing compensation model is derived by modeling the mechanism of generation of shadowing effects, i.e., modeling the image intensities in both the absence and presence of shadowing. This takes the following form:

$$
\mathrm{BSE}_{\text {shadowless }}=\mathrm{BSE} /\left(\sum_{i=S+1}^{K-S} \frac{1-\sin ^{n+1} \theta_{i}}{K-2 S}\right),
$$

with the thresholding operation

$$
\theta_{i}=\left\{\begin{array}{ll}
0 & \theta_{i} \leq \theta_{\min }, \\
\theta_{\max } & \theta_{i} \geq \theta_{\max }, \\
\theta_{i} & \text { otherwise. }
\end{array} \quad S+1 \leq i \leq K-S .\right.
$$

Here BSE $\mathrm{B}_{\text {shadowless }}$ and BSE denote the shadowless and observed image intensities, respectively, $K$ represents the number of segments of the range of the azimuth angle, $\theta_{i}$ describes the shadowing angle in the $i^{\text {th }}$ segment, the integer $S=\left[\frac{\phi_{\min }}{\pi / K}\right]$, wherein "[ ]" denotes the rounding operator, and $\phi_{\min }, \theta_{\min }, \theta_{\max }$, and $n$ are parameters.

In the theoretical derivation of the shadowing compensation model, we assumed that shadowing effects exist only in horizontal regions (as shown in Fig. 2 (a)). Because of this simplification, the shadowing compensation model takes a compact explicit form that is convenient for applications. However, the model would incur large errors if it were applied to shadowing regions on surfaces having very large inclinations (as shown in Fig. 2 (b)). Therefore, to increase the accuracy on general inclined surfaces and extend the scope of the model, we abandon the restrictive assumption and extend the model from horizontal surfaces to more general inclined surfaces.

\section{Improvements}

Although the modeling procedure of the extended shadowing compensation model is analogous to that of the previous model, the mathematical derivation can be much more complicated. The derivation of the extended model is based on the following assumptions:

1. The BSE image intensity is proportional to the amount of backscattered electrons (BSEs) collected by the detector.

2. The angle distribution of BSEs can be expressed as follows:

$$
\frac{d \eta}{d \omega}=\frac{\eta_{0}}{\pi}(\cos \xi)^{n}
$$




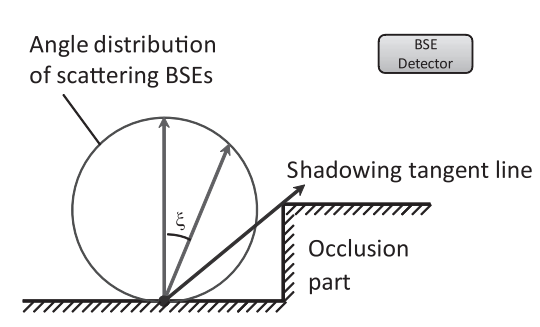

Fig. 2: Shadowing effects. BSEs emitted in a direction below the shadowing tangent line are generally absorbed by the occlusion region and consequently are not collected by the detector. (a) Shadowing on the horizontal surface. (b) Shadowing on the inclined surface

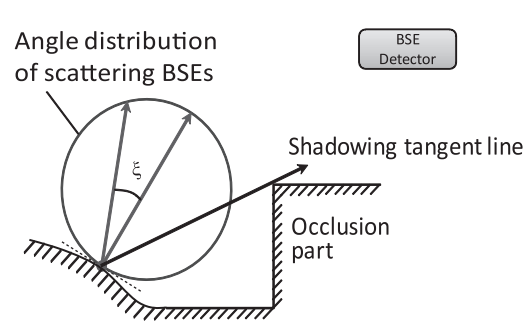

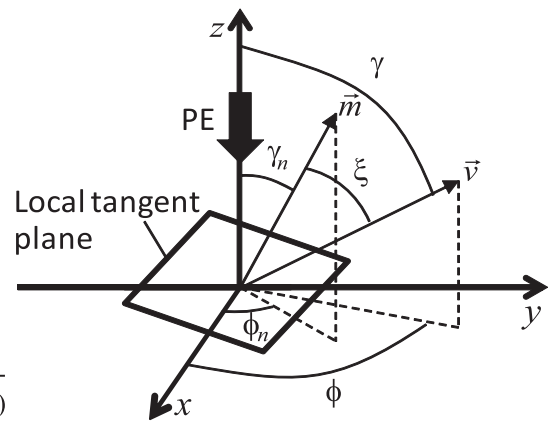

Fig. 3: Coordinate system where $\xi$ describes the angle between the surface normal and the direction of the electron emission; $\eta_{0}$ is a coefficient; $\eta$ and $\omega$ denote the quantity of BSEs and the solid angle, respectively; and the parameter $n$ ensures more flexibility while describing the angular distribution in terms of the Lambert's cosine law, in which the parameter is equal to one ${ }^{14)}$.

\subsection{Coordinate System}

Fig. 3 shows the coordinate system for 3D measurements. The $z$-axis is coincident with the incident primary electron (PE) beam, the $x$-axis is the direction along which two detectors are positioned, and the $y$-axis is perpendicular to both the $x$ and $z$ axes according to the right-hand rule. The surface of the measuring specimen can be represented as $z(x, y)$. The normal to the tangent plane of the surface is $\vec{m}=\left(-\frac{\partial z}{\partial x},-\frac{\partial z}{\partial y}, 1\right)$. For an arbitrary emitting direction of BSEs $\vec{v}=(\cos \phi \sin \gamma, \sin \phi \sin \gamma, \cos \gamma)$, the angle distribution takes the following form:

$$
\frac{d \eta}{d \omega}=\frac{\eta_{0}}{\pi}\left(\frac{\cos \gamma-\frac{\partial z}{\partial x} \cos \phi \sin \gamma-\frac{\partial z}{\partial y} \sin \phi \sin \gamma}{\sqrt{1+\left(\frac{\partial z}{\partial x}\right)^{2}+\left(\frac{\partial z}{\partial y}\right)^{2}}}\right)^{n},
$$

where $\gamma$ and $\phi$ denote the zenith and azimuth angles, respectively.

\subsection{BSE Intensity in the Absence of Shadowing}

In the non-shadowing case, the detectable range of the azimuth angle $\phi$ for detectors should ideally be $-\frac{\pi}{2} \leq \phi \leq \frac{\pi}{2}$. Nonetheless, BSEs in the region where $\phi$ is near $\pm \frac{\pi}{2}$ generally cannot be detected owing to the practical limitations of the detector. Therefore, we introduce the parameter $\phi_{\min }$ to model this shortfall ${ }^{11)}$; then the range of the azimuth angle in the practical detectable region should be $-\frac{\pi}{2}+\phi_{\min } \leq \phi \leq \frac{\pi}{2}-\phi_{\min }$.

The range of the zenith angle $\gamma$ is more complicated. As shown in Fig. 4, different inclination conditions of the surface give rise to different expressions for the upper limit. In the case of Fig. 4 (a), the upper limit of the zenith angle $\gamma$ is up to the surface tangent direction $\vec{t}=\left(\cos \phi, \sin \phi, \cos \phi \frac{\partial z}{\partial x}+\sin \phi \frac{\partial z}{\partial y}\right)$, i.e., the intersection of the tangent plane and the zenith integration plane (see also Fig. 4 (c)), which takes the following form:

$$
\gamma_{\vec{t}}(\phi)=\arccos \left(\frac{M}{\sqrt{1+M^{2}}}\right)
$$

where $M=\cos \phi \frac{\partial z}{\partial x}+\sin \phi \frac{\partial z}{\partial y}$.

In the case of Fig. 4 (b), the surface tangent direction is below the horizontal direction. However, the BSE detector can only ideally collect BSEs above the horizontal direction. The electrons with emission angles below the horizontal direction generally cannot be detected. Furthermore, because of the finite size of practical detectors, electrons with an emission direction near the horizontal plane may not be collected. Another parameter $\theta_{\min }$ is introduced to model such practical factors ${ }^{11)}$. Therefore, in this case, the upper limit of the zenith angle cannot be up to the surface tangent direction, but only up to $\frac{\pi}{2}-\theta_{\min }$.

Summarizing the different cases, the upper limit of the zenith angle should be $\gamma(\phi)=\min \left\{\frac{\pi}{2}-\theta_{\min }, \gamma_{\vec{t}}(\phi)\right\}$, and the detectable region $\Omega$ in the case of non-shadowing can take the following form:

$$
\Omega:\left\{\begin{array}{l}
-\frac{\pi}{2}+\phi_{\min } \leq \phi \leq \frac{\pi}{2}-\phi_{\min } \\
0 \leq \gamma \leq \min \left\{\frac{\pi}{2}-\theta_{\min }, \gamma_{\vec{t}}(\phi)\right\}
\end{array}\right.
$$

The image intensity (signal output) on each detector is proportional to the number of collected electrons, which can be modeled by the following double integral:

$$
\mathrm{BSE}_{\text {shadowless }}=\iint_{\Omega} \frac{\eta_{0}}{\pi}(\cos \xi)^{n} d \omega=\frac{\eta_{0}}{\pi} \iint_{\Omega} I(\phi, \gamma) d \phi d \gamma,
$$

where the integrand ${ }^{1}$

$$
I(\phi, \gamma)=\left(\frac{\cos \gamma-\frac{\partial z}{\partial x} \cos \phi \sin \gamma-\frac{\partial z}{\partial y} \sin \phi \sin \gamma}{\sqrt{1+\left(\frac{\partial z}{\partial x}\right)^{2}+\left(\frac{\partial z}{\partial y}\right)^{2}}}\right)^{n} \sin \gamma
$$

Unfortunately, this integral cannot be evaluated via elementary functions.

\subsection{BSE Intensity in the Presence of Shadowing}

In the shadowing case, the azimuth range of the detectable region should be identical to that of the non-shadowing case. However, the zenith range is generally smaller because of occlusion. As shown in Fig. 5, the portion of the BSEs from the shadowing tangent line to the local surface tangent direction is occluded and generally cannot be collected by the corresponding detector. The range of the zenith angle should be $0 \leq \gamma \leq \gamma_{\vec{t}}(\phi)-\theta(\phi)$, where $\theta(\phi)$ is the shadowing angle defined as the angle between the shadowing tangent line and surface tangent direction. Nevertheless, it is likely that the shadowing tangent direction is below the lowest

\footnotetext{
${ }^{1}$ More rigorously, the integrand should be represented as $I\left(\phi, \gamma, \frac{\partial z}{\partial x}, \frac{\partial z}{\partial y}\right)$, and consequently the image intensity in the nonshadowing case would be $\operatorname{BSE}_{\text {shadowless }}\left(\frac{\partial z}{\partial x}, \frac{\partial z}{\partial y}\right)$, which would be differing corresponding to the inclination condition of the local tangent plane.
} 


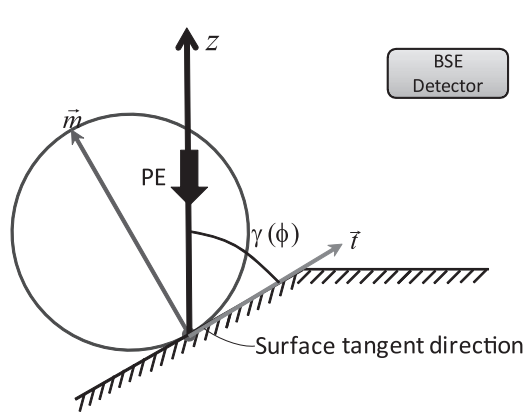

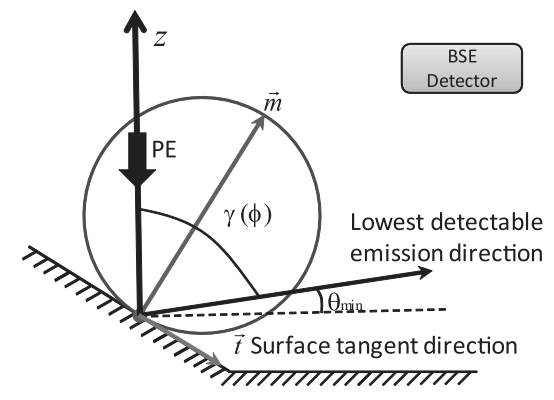

Fig. 4: Zenith ranges in the absence of shadowing. (a) The upper limit of the zenith angle is up to the surface tangent direction. (b) The upper limit of the

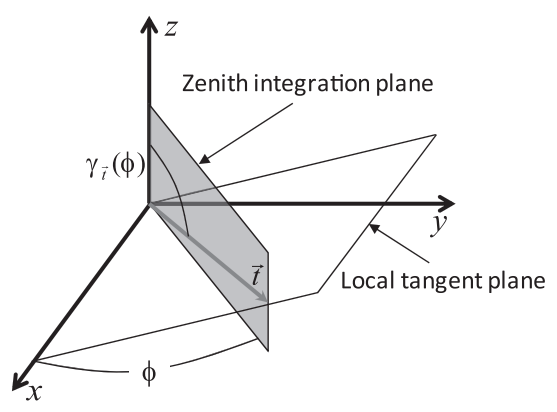

(c) zenith angle can be only up to the lowest detectable emission direction, electrons below this direction generally cannot be collected by a detector. (c) One case of upper limit of zenith angle

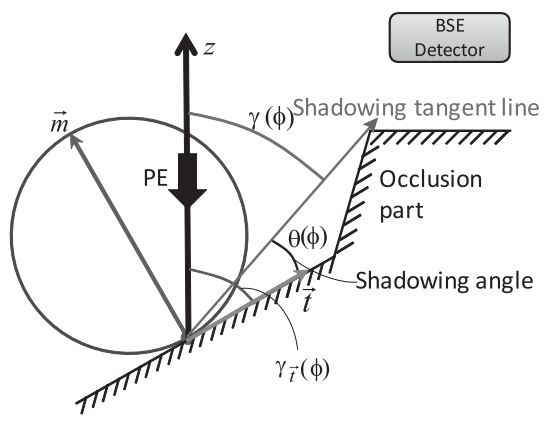

Fig. 5: Zenith ranges in the presence of shadowing. (a) The upper limit of the zenith angle is up to the shadowing tangent line. (b) The upper limit of the

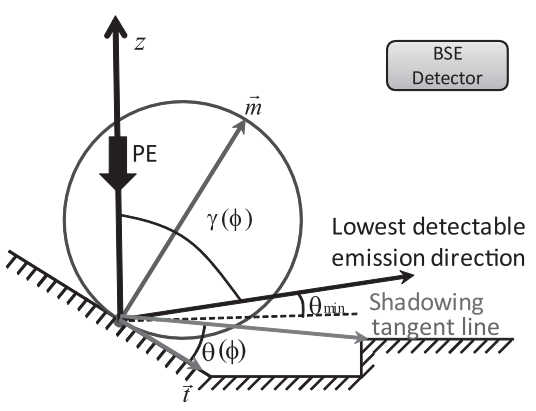

Fig. 5: Zenith ranges in the presence of shadowing. (a) The upper limit of the zenith angle is up to the shadowing tangent line. (b) The upper limit of the

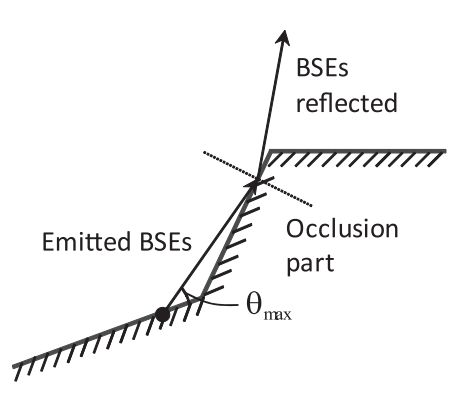

(c) zenith angle can only be up to the lowest detectable emission direction; electrons below this direction generally cannot be collected by a detector, which indicates no shadowing in fact. (c) Illustration of the practical factor modeled by $\theta_{\max }$

detectable emission direction, i.e., $\gamma_{\vec{t}}(\phi)-\theta(\phi)>\frac{\pi}{2}-\theta(\phi)$. So, in fact, there is no shadowing in the $\phi$ direction (see also Fig. 5 (b)). Including both cases for the zenith range, the upper limit of the zenith angle should be $\gamma(\phi)=\min \left\{\frac{\pi}{2}-\theta_{\min }, \gamma_{\vec{t}}(\phi)-\theta(\phi)\right\}$. Therefore, the detectable region should take the following form:

$$
\Omega^{S}:\left\{\begin{array}{l}
-\frac{\pi}{2}+\phi_{\min } \leq \phi \leq \frac{\pi}{2}-\phi_{\min } \\
0 \leq \gamma \leq \min \left\{\frac{\pi}{2}-\theta_{\min }, \gamma_{\vec{t}}(\phi)-\theta(\phi)\right\}
\end{array}\right.
$$

However, to optimize the process for modeling shadowing effects, a practical factor must be considered. As illustrated in Fig. 5 (c), when the emission direction of the BSEs is nearly parallel to the slope of the shadowing object, electrons are likely to be reflected rather than absorbed. We therefore simply set a threshold, $\theta_{\max }$, for the shadowing angle. When the shadowing angle is larger than $\theta_{\max }$, it is set to $\theta_{\max }$.

Similar to the case of absence of shadowing, the observed image intensity can also be modeled by the following double integral:

$$
\mathrm{BSE}=\iint_{\Omega^{S}} \frac{\eta_{0}}{\pi}(\cos \xi)^{n} d \omega=\frac{\eta_{0}}{\pi} \iint_{\Omega^{S}} I(\phi, \gamma) d \phi d \gamma .
$$

Here the integrand $I(\phi, \gamma)$ takes the same form as (7). This integral cannot be evaluated via elementary functions.

\subsection{Extended Shadowing Compensation Model}

Equation (8) models the observed image intensity that may include the presence of shadowing effects. In fact, the shadowless case can be considered to be a special shadowing case in which the shadowing angle $\theta(\phi)=0$. Generalizing this observation, we can deduce the following extended shadowing compensation model by combining (6) and (8).

$$
\mathrm{BSE}_{\text {shadowless }}=R \cdot \mathrm{BSE} \text {. }
$$

Here the compensation ratio $R$ can be expressed as

$$
R=\frac{\iint_{\Omega} I(\phi, \gamma) d \phi d \gamma}{\iint_{\Omega^{S}} I(\phi, \gamma) d \phi d \gamma}
$$

Unlike the horizontal case, the compensation ratio (10) cannot be evaluated explicitly.

\subsection{Numerical Solution}

Since the two integral in (10) cannot be evaluated explicitly, the extended shadowing compensation model cannot take an explicit form. Therefore, we must explore a numerical solution for the model.

For a uniform discretization of the domain of integration (detectable region), we can simply merge the two detectable regions $\Omega$ and $\Omega^{s}$ into a quarter sphere $\Omega_{Q}:-\frac{\pi}{2} \leq \phi \leq \frac{\pi}{2}, 0 \leq \gamma \leq \frac{\pi}{2}$, as both are subsets of $\Omega_{Q}$, i.e., $\Omega, \Omega^{s} \subseteq \Omega_{Q}$. $\Omega_{Q}$ can be discretized into $K \times L$ elementary regions with the azimuth step $\Delta \phi$ and the zenith step $\Delta \gamma$, see Fig. 6. The step sizes of $\Delta \phi$ and $\Delta \gamma$ should be set sufficiently small to ensure accuracy. The detectable region $\left(\Omega\right.$ or $\left.\Omega^{s}\right)$ can be regularized in the following way:

(1) $\phi_{\min } \approx S \cdot \Delta \phi$, where $S=\left[\frac{\phi_{\min }}{\Delta \phi}\right]$. The azimuth range can be approximated as $\phi_{S} \leq \phi \leq \phi_{K-S}$.

(2) $\gamma(\phi) \approx J_{i} \cdot \Delta \gamma$ for $\phi_{i-1} \leq \phi \leq \phi_{i}, S+1 \leq i \leq K-$ 


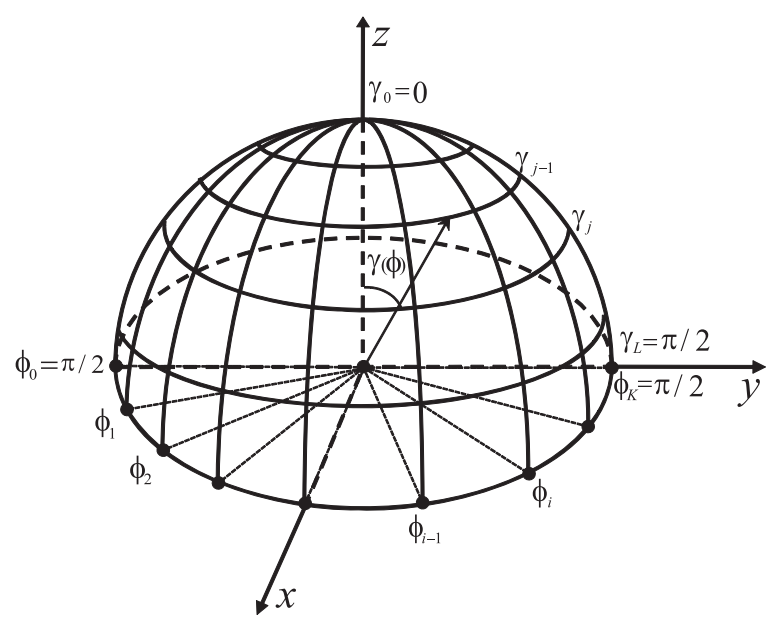

(a) Discretization shown in 3D

Regularized upper limit of zenith angle

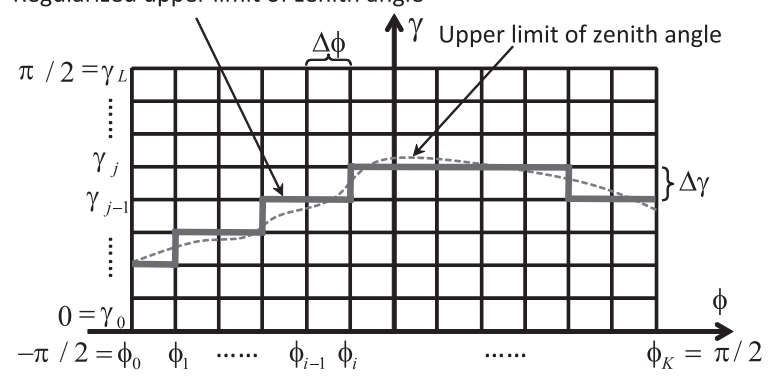

(b) Discretization shown in 2D

Fig. 6: Discretization of the detectable region. (a) Illustration of discretization of $\Omega_{Q}$ shown in 3D space. (b) Illustration of discretization of $\Omega_{Q}$ shown in $2 \mathrm{D}$ space and regularization of detectable region

$S$. Here $J_{i}=\left[\frac{\gamma\left(\phi_{i-1}+\Delta \phi / 2\right)}{\Delta \gamma}\right]$. The zenith range can be approximated as $0=\gamma_{0} \leq \gamma \leq \gamma_{J_{i}}$, for $S+1 \leq i \leq K-S$, see Fig. 6 (b).

Therefore, the regularized detectable region $\left(\Omega\right.$ or $\Omega^{s}$ ) can be expressed as follows:

$$
\Omega / \Omega^{S}:\left\{\begin{array}{l}
\phi_{S} \leq \phi \leq \phi_{K-S} \\
\gamma_{0} \leq \gamma \leq \gamma_{J_{i}}, \quad S+1 \leq i \leq K-S
\end{array}\right.
$$

The integrand within each elementary rectangular region $\left(\phi_{i-1} \leq \phi \leq \phi_{i}, \gamma_{j-1} \leq \gamma \leq \gamma_{j}\right.$ for $S+1 \leq i \leq K-S$, $\left.1 \leq j \leq J_{i}\right)$ can be approximated as $I(\phi, \gamma) \approx I_{i j}=I\left(\phi_{i-1}+\right.$ $\left.\Delta \phi / 2, \gamma_{j-1}+\Delta \gamma / 2\right)$.

With these approximations, we can ultimately achieve numerical solutions for the two double integrals:

$$
\iint_{\Omega / \Omega^{S}} I(\phi, \gamma) d \phi d \gamma \approx \sum_{i=S+1}^{K-S} \sum_{j=1}^{J_{i}} I_{i j} \Delta \phi \Delta \gamma
$$

Consequently, the compensation ratio (10) can be numerically calculated with this numerical method.

\section{Iterative Shadowing Compensation Method}

In order to deal with shadowing errors in the SEM photometric stereo method, it is reasonable to eliminate shadowing effects in

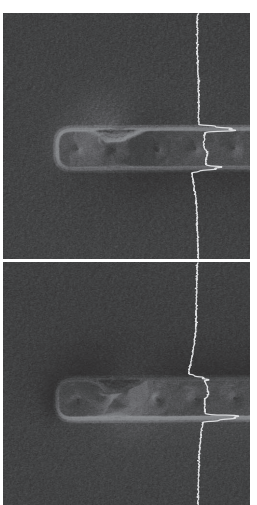

Fig. 7: Comparison between the original and compensated images of the

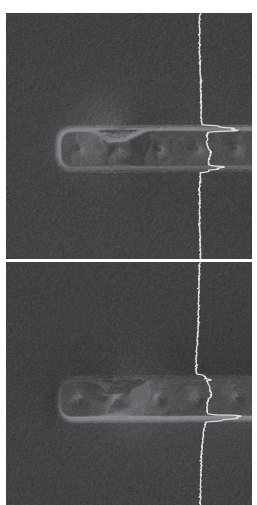

Fig. 7: Comparison between the original and compensated images of the

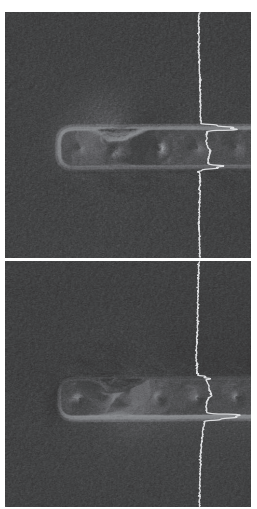

Fig. 7: Comparison between the original and compensated images of the "semi-line" pattern: (a) original left (upper) and right (lower) images, (b) compensated left (upper) and right (lower) images via SCM, and (c) compensated left (upper) and right (lower) images via ESCM

the images $(B L$ and $B R)$ by revising the observed image intensities using the derived extended shadowing compensation model. Once the shadowless image pair is obtained, an accurate 3D shape can be reconstructed by applying Suganuma's equation (gradient calculation) and depth from gradient method in turn. However, the true compensation ratio can only be calculated from the true $3 \mathrm{D}$ shape, which is, in fact, the target we are pursuing. The problem essentially boils down to something such as a "chicken-and-egg" dilemma, that the underlying shadowless images can be estimated from 3D shape via the extended shadowing compensation model, while the 3D shape can be reconstructed from shadowless images. In order to solve this problem, the present authors have proposed the iterative shadowing compensation method to conduct the shadowing compensation in an iterative scheme:

\section{Iterative Shadowing Compensation Algorithm}

S1. Reconstruction of the $3 \mathrm{D}$ surface $z^{0}$ from the original images $B L$ and $B R$.

$\mathrm{S} 2$. At the $k^{\text {th }}$ iteration, the compensation ratios are calculated from the reconstructed surface $z^{k-1}$ from the previous iteration. Using extended shadowing compensation model to estimate shadowless images $B L_{c}^{k}$ and $B R_{c}^{k}$.

S3. A new approximation of the real surface $z^{k}$ is reconstructed from $B L_{c}^{k}$ and $B R_{c}^{k}$.

S4. If the difference between $z^{k}$ and $z^{k-1}$ is smaller than the given threshold, the algorithm will be terminated; otherwise, the iteration will be repeated.

The calculated compensation ratios in S2 are not the actual ones, but approximations, which can modified the images at some extent.

After convergence of the algorithm, both the reconstructed 3D surface and compensated shadowless images are obtained.

\section{Experimental Results}

In this section, we present the results of experiments on real image data and examine the robustness and effectiveness of the proposed method under shadowing conditions. Two different patterns 


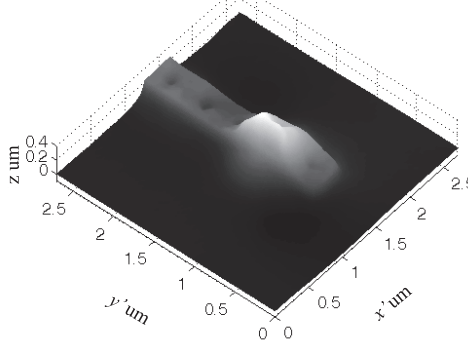

(a)

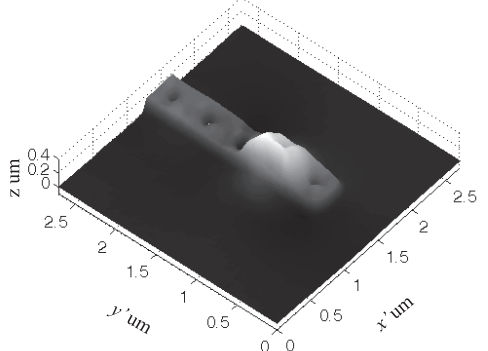

Fig. 8: Comparison of three-dimensional surfaces and section profiles of the "semi-line" pattern: (a) 3D surface before compensation, (b) 3D surface after

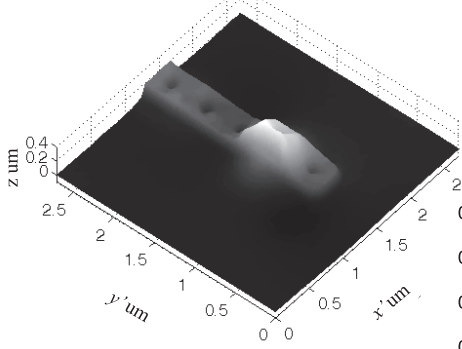

(b)

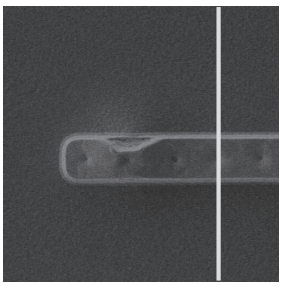

(d)

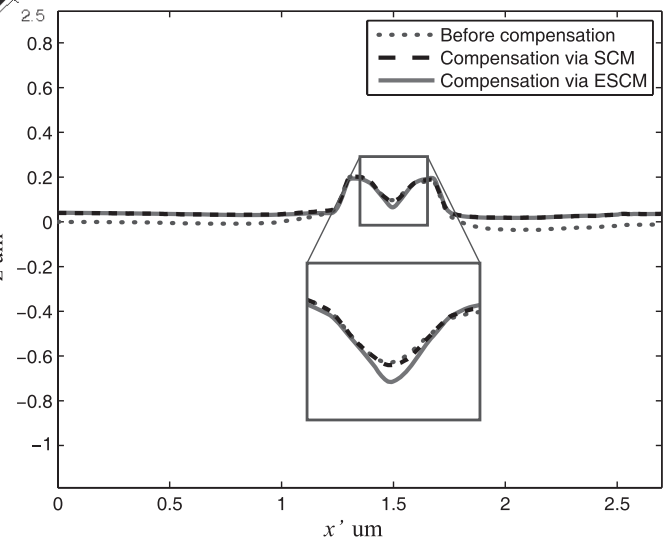

(e) compensation via SCM, (c) 3D surface after compensation via ESCM, (d) selected section profile position marked on the original left image and (e) section profiles at position marked in (d)

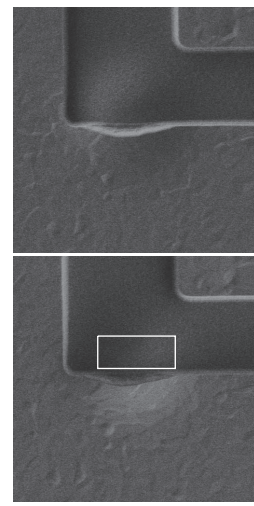

Fig. 9: Comparison between the original and compensated images of the

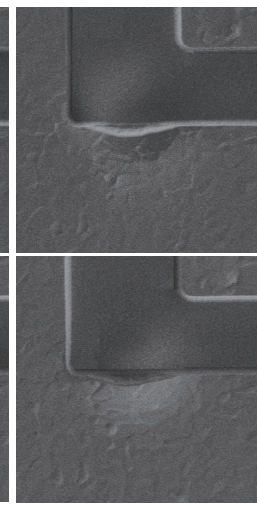

Fig. 9: Comparison between the original and compensated images of the

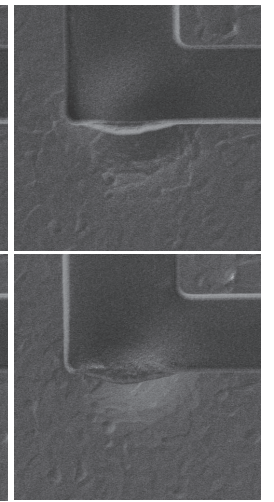

Fig. 9: Comparison between the original and compensated images of the "concave" pattern: (a) original left (upper) and right (lower) images, marked region of interest (ROI) for quantitative analysis, (b) compensated left (upper) and right (lower) images via SCM, and (c) compensated left (upper) and right (lower) images via ESCM

of semiconductor devices with strong shadowing effects existing on both flat regions and inclined regions were used for evaluating the performance of the proposed extended shadowing compensation model (ESCM) and the previous shadowing compensation model (SCM). Both devices had embedded defects, which included a "semi-line" pattern and a "concave" pattern. The SEM used in our experiment performs raster scanning along the direction located $45^{\circ}$ from the $x$-axis. (The $x$-axis is defined as the direction along which the detectors are positioned.) Therefore, the $x^{\prime}$-axis and the $y^{\prime}$-axis consistent with the image array are defined for convenience in displaying results.

Fig. 7 and Fig. 8 show the experimental results for the "semiline" pattern. Fig. 7 (a) shows the original left and right images. It is obvious that the lower part near the "semi-line" in the original left image and the upper and upper left part near the "semi-line" in

the original right image are notably dark, which is, in fact, owing to shadowing effects. Such shadowing effects can be more clearly demonstrated using average image intensity plots (the average is used to avoid submersion of the plot into noise). In this case, the "semi-line" part plays the role of an occlusion part. Such shadowing errors in image intensities give rise to significant departures of the gradient measurements from the true ones (zeros at ideally flat regions); this is reflected in the reconstructed 3D surface as a "rising slope" toward the "semi-line" part (see the 3D surface in Fig. 8 (a) and section profiles in Fig. 8 (e)). Besides shadowing effects in the horizontal area around the "semi-line" part, strong shadowing effects are also present in the dimple regions inside the "semi-line" part, where the surface is not horizontal but inclined.

Fig. 7 (b) and Fig. 8 (b) show the compensated images and the 3D surface of the "semi-line" pattern via SCM, and Fig. 7 (c) and Fig. 8 (c) show the compensated images and 3D surface of the "semi-line" pattern via ESCM, respectively. Both ESCM and SCM achieve proper performance of shadowing compensation in the horizontal shadowing region around the "semi-line" part. In the compensated left and right images, the brightness of the flat regions around the "semi-line" part demonstrates a certain uniformity with that in the other flat regions without shadowing effects. The corresponding average intensity plots also highlight suitabe compensation of the shadowing errors in the flat regions. Moreover, the reconstructed 3D surface shape and section profiles also show large modifications (see Fig. 8 (b), (c), and (e)) in the "rising slope" regions on both sides of the "semi-line, "where the shape is properly corrected to a nearly flat level, as it should be.

However, ESCM can perform even better in the shadowing region of inclined surfaces, as shown in the dimple regions. As shown in Fig. 8 (e) (since the dimple part is too small to give a vivid view, the figure in that part is magnified), the compensated section 


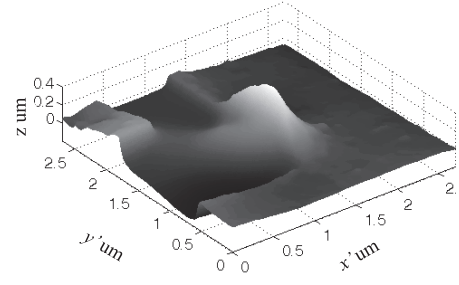

(a)

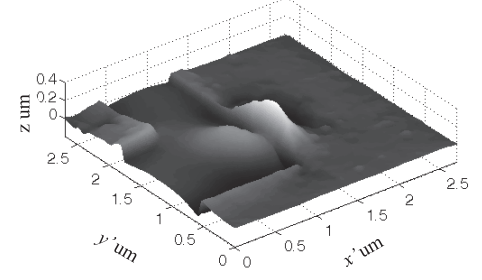

(c)

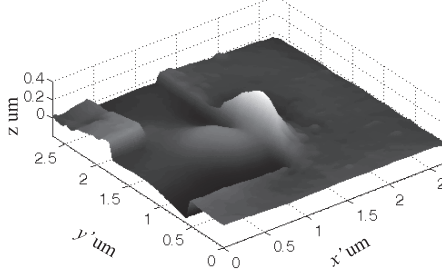

(b)

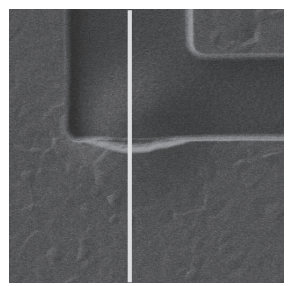

(d)

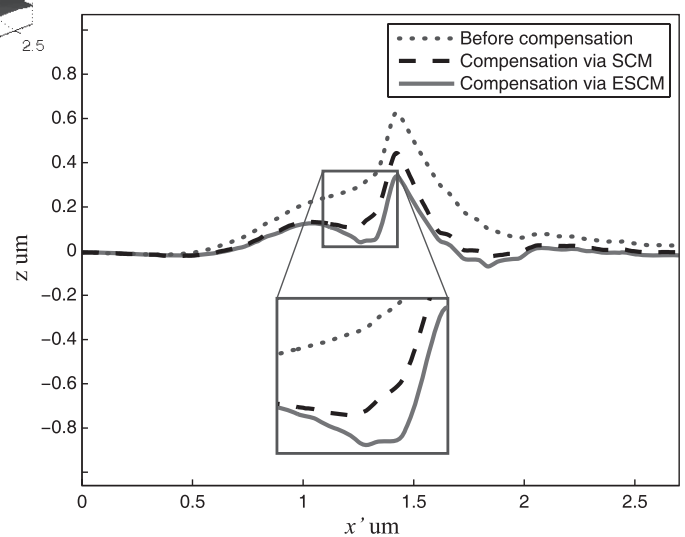

(e)

Fig. 10: Comparison of three-dimensional surfaces and section profiles of the "concave" pattern: (a) 3D surface before compensation, (b) 3D surface after compensation via SCM, (c) 3D surface after compensation via ESCM, (d) selected section profile position marked on the original left image and (e) section profiles at position marked in (d)

profile within the dimple part via SCM shows little modification, which nearly coincides with the profile before compensation. But the compensation profile of ESCM reveals large modifications, indicating that ESCM may perform better on surfaces having large inclinations.

Fig. 9 and Fig. 10 show experimental results for the "concave" pattern. The embedded defect caused a very high "peak" part in the semiconductor device. Therefore, the shadowing effects on both sides of the defect "peak" are quite strong. Since the "peak" part is very high and plays the significant role of an occluding part, it causes a "rising slope" effect, that is analogous to the previous "semi-line" case. Note that the shadowing regions are not horizontal, but have large inclinations. Although the inclinations are not readily distinguishable by the image brightness, they can be clearly observed in the 3D shape and the corresponding section profile.

Fig. 9 (b) and Fig. 10 (b) show the compensated images and 3D surface of the "concave" pattern via SCM, and Fig. 9 (c) and Fig. 10 (c) show the compensated images and 3D surface of the "concave" pattern via ESCM. As vividly demonstrated in Fig. 10 (e), the compensated shape of the defect part (shadowing effect on the inclined surface) via ESCM reveals better modification than that via SCM, although SCM also shows large modifications. The defect part in the compensated 3D shape in Fig. 10 (c) becomes more distinguishable and recognizable. The experimental results in turn indicate that compared with SEM, ESCM can generally achieve comparative performance in horizontal regions and even better performance on inclined surfaces.

In the experiments, we use the difference of reconstructed surface between two successive iterations to reflect the convergence property of the iterative shadowing compensation algorithm, given by $e^{k}=\frac{1}{M N} \sqrt{\sum_{i=1}^{M} \sum_{j=1}^{N}\left(\frac{z^{k}(i, j)-z^{k-1}(i, j)}{h}\right)^{2}}$. Here $k$ rep-

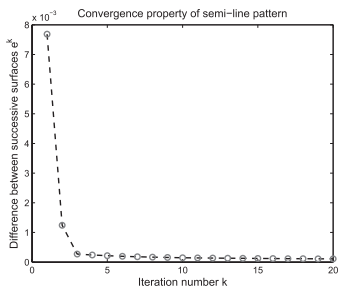

Fig. 11: Convergence processes of the iterative shadowing compensation

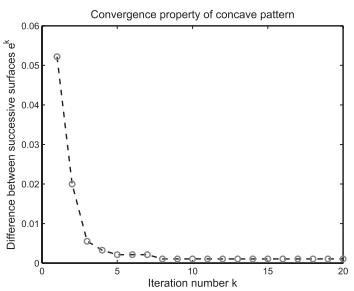

Fig. 11: Convergence processes of the iterative shadowing compensation algorithm. (a) "Semi-line" pattern. (b) "Concave" pattern

resents iterative step index, $i$ and $j$ refer to image row and column indices, $M$ and $N$ denote image height and width, and $h$ is pixel size to make the metric $e^{k}$ dimensionless. Fig. 11 (a) and (b) show the convergence processes of semi-line pattern and concave pattern, respectively, where the iteration number is set to 20 . As observed in both cases, the method can converge quickly. In practice, we will set a threshold value $T$, say $T=10^{-3}$, to terminate the algorithm earlier if $e^{k}<T$ at some step $k$.

A high speed implementation of our method has not yet been examined. The average computing time per each step with image size $512 \times 512$ is, currently, about 67.3 seconds in $\mathrm{C}++$ on a $2.93 \mathrm{GHz}$ PC with $4 \mathrm{G}$ memory. However, we realize that it should be as a future work to improve the computational efficiency by means of exploring efficient numerical methods (such as nonlinear multigrid method ${ }^{15)}$ ) or parallel computing (GPU implementation, for instance).

For a quantitative assessment of our method, the most straightforward way should be comparing reconstruction results to the corresponding actual ones. However, it is generally difficult to obtained the height of defect parts. Because of lack of ground truth data, we alternatively adopt a somewhat indirect evaluation method. We manually select a region of interest (ROI) in the right 


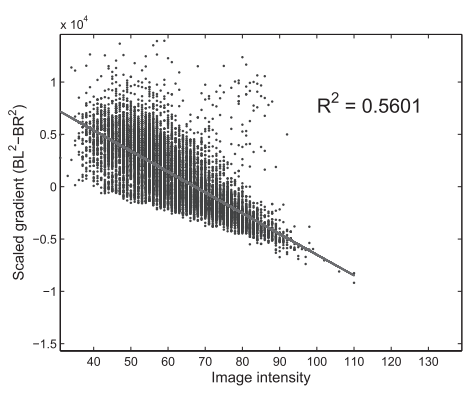

(a)

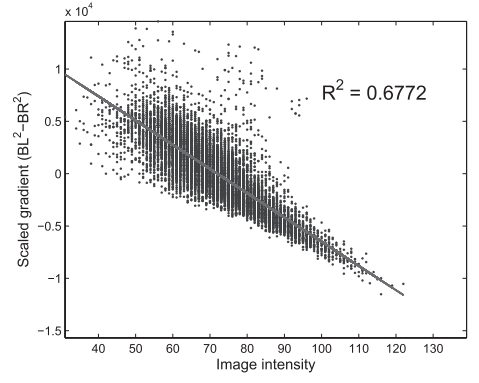

(b)

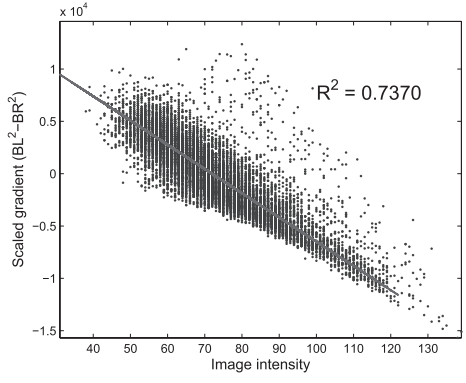

(c)

Fig. 12: Quantitative evaluation of shadowing compensation by compactness between image intensity and gradient within the ROI marked in Fig. 9 (a): (a) before compensation, (b) compensation via SCM, and (c) compensation via ESCM

image of the "concave" pattern marked in Fig. 9 (a), where the region contains strong shadow on the curved surface. Ideally, the image intensity in the ROI should have a one-to-one mapping to the corresponding gradient value of the surface if the intensity is ideally correct (without noise or shadowing errors). Because, from the physical imaging point of view, each point with a distinct inclination (gradient) on the surface gives rise to the corresponding unique and distinct intensity value. However, as shown in Fig. 12 (a) (Here we scale the gradient value by ignoring the constant parts in Suganuma's equation (1) for convenience.), such uniqueness will not hold when the intensity is polluted by random signal noise or shadowing errors. Our idea is that the data should be more compact to be closer to the one-to-one mapping if the shadowing errors are properly eliminated or reduced. The difficulty is that the mapping is related to the shape of the surface and thus unknown. We ultimate employ the linear model based on the observation that the data seemly obey a linear relationship. Moreover, on account of high noise level, we prefer a simpler model in general. The straight lines in Fig. 12 are the linear regression results and $R^{2}$ denotes the square of correlation coefficient which indicates the compactness. Compared with Fig. 12 (b) (compensated data via SCM), the data in Fig. 12 (c) (compensated data via ESCM) tend to be more compact which implies the shadowing errors are reduced in a more proper way via ESCM.

\section{Conclusion and Future Work}

In this paper, we have described an extension of the shadowing compensation model from restricted horizontal surfaces to more general inclined surfaces. The new model is derived by modeling image intensities in both the absence and presence of shadowing on general inclined surfaces. The new model does not include the restrictive assumption that shadowing effects exist only on horizontal surfaces. The experimental results show that the extended model, compared to the previous one, can achieve comparable performance on horizontal regions and even better performance on inclined surfaces.

Future studies designed to validate the feasibility of this approach in real applications are under consideration. For example, the proposed method should be evaluated on various semiconductor patterns in actual production lines with different processes and layouts. Moreover, we will make some ground truth data, for instance by atomic force microscopy (AFM), for further investiga- tion of the performance of our method.

\section{References}

1) A. Miyamoto and T. Honda: Development of Surface/Embedded Particle Classification Algorithm for SEM-ADC, IEICE Transaction on Information \& Systems, J91-D, 6, (2008), 1604.

2) T. Oshima, S. Kimoto and T. Suganuma: Stereomicrography with a Scanning Electron Microscope, Photogrammetric Engineering, 36, (1970), 874.

3) G. Koenig, W. Nickel, J. Storl, D. Meyer, and J. Stange: Digital Stereophotogrammetry for Processing SEM Data, Scanning, 9, (1987), 185.

4) O. V. Kholodilov, A. Ya. Grigoryev and N. K. Myshkin: Reconstruction of true topographies of solid surfaces in scanning electron microscopes using secondary electrons, Scanning, 9, (1987), 156.

5) J. Lebiedzik: An Automatic Topographical Surface Reconstruction in the SEM, Scanning, 2, (1979), 230.

6) I. C. Carlsen: Reconstruction of True Surface-Topographies in Scanning Electron Microscopes Using Backscattered Electrons, Scanning, 7, (1985), 169.

7) T. Suganuma: Measurement of Surface Topography Using SEM with two Secondary Electron Detectors, Journal of Electron Microscopy, 34, 4, (1985), 328.

8) E. Reithmeier, T. Vynnyk and T. Schultheis: 3D-measurement using a scanning electron microscope, Applied Mathematics and Computation, 217, (2010), 1193.

9) J. Paluszyński and W. Slówko: Surface Reconstruction with the Photometric Method in SEM, Vacuum, 78, (2005), 533.

10) J. Paluszyński and W. Slówko: Compensation of the Shadowing Error in Three-dimensional Imaging with a Multiple Detector Scanning Electron Microscope, Scanning, 224, (2006), 93.

11) D. Chen, A. Miyamoto and S. Kaneko: Robust Surface Reconstruction in SEM with Two BSE Detectors, Proceeding of Mecatronics REM, (2012), 64.

12) A. Agrawal, R. Raskar and R. Chellappa: What is the Range of Surface Reconstructions from a Gradient Field, Proceeding of ECCV, (2006), 578.

13) B. K. P. Horn and M. J. Brooks: The Variational Approach to Shape from Shading, Computer Vision, Graphics and Image Processing, 33, (1986), 174.

14) L. Reimer: Scanning Electron Microscopy: Physics of Image Formation and Microanalysis, 2nd ed. Springer, (2008), 146.

15) A. Bruhn, J. Weickert, T. Kohlberger, C. Schnorr: A multigrid platform for real-time motion computation with discontinuity-preserving variational methods, International Journal of Computer Vision, 70, 3, (2006), 257. 